\title{
BALSA DEL TRASVASE ESLA-CARRIÓN. SU IMPORTANCIA EN EL DESARROLLO RURAL REGIONAL Y UN EJEMPLO DE REGULACIÓN DE RECURSOS Y COLABORACIÓN ENTRE LA ADMINISTRACIÓN Y LAS COMUNIDADES DE REGANTES
}

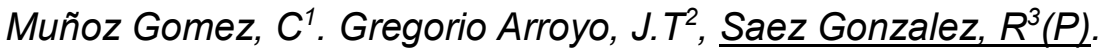

\author{
${ }^{1}$ Ingeniera Civil. Tecnico. ITACyL. \\ ${ }^{2}$ Ingeniero de Caminos, Canales y Puertos. Jefe de Unidad de Obras. ITACyL \\ ${ }^{3}$ Ingeniero Agrónomo. Subdirector de Infraestructuras. ITACyL
}

\section{Resumen}

Se describe la actuación de transformación de regadío en una zona de la Tierra de Campos de las provincias de Palencia y Valladolid, para lo cual ha sido necesario implementar una solución de regulación de recursos hídricos de invierno, mediante una balsa de $10 \mathrm{Hm}^{3}$ de materiales sueltos de la zona, que permite poner en regadío más de 2.000 hectáreas en una zona alejada de los cursos de agua regulados. Para ello se emplea como elemento de transporte el Canal de Transvase Esla Carrión, infraestructura que no tiene uso en invierno cuando hay que derivar los recursos. Es una forma de aumentar la capacidad de regulación en una cuenca como la del Duero que es la que peor capacidad de regulación tiene en España, respecto a sus aportaciones. Se describe asimismo los requerimientos en materia de seguridad que una infraestructura como esta tiene, y la fórmula que hay en Castilla y León desarrollar estas actuaciones mediante una colaboración entre el ITACyL y las Comunidades de Regantes.

\begin{abstract}
"Abstract"
The irrigation transformation project in the area of Tierra de Campos (Palencia and Valladolid provinces) is described. The project implemented a regulation reservoir to store water resources from Winter time. The reservoir has a volume of $10 \mathrm{Hm} 3$ and is built in a flat area with embarkment dams with materials from the surroundings. The irrigated area covered by the reservoir is larger than 2,000 hectares. The reservoir is located far away from main rivers so it is filled using the Esla Carrión Transfer Canal. This canal has no use during winter when resources must be diverted. This project represents a way of increasing the regulatory capacity in the Duero river basin. This basin is the one with the lowest regulation/contributions ratio within Spain. The paper also describes the security requirements of the infrastructure, and the formula that exists in Castilla y León to develop these actions through a collaboration between the regional government by means of ITACyL and the Irrigation Districts.
\end{abstract}

\section{1.- Introducción. Objetivo del Trabajo.}

El objetivo de este trabajo es describir la planificación y ejecución de las infraestructuras necesarias para la transformación en regadío de una zona de 2.136 hectáreas en un sector ubicado en el corazón de la denominada Tierra de Campos en las provincias de Palencia y Valladolid, dotándola de una regulación característica, mediante una balsa fuera de cauce de $10 \mathrm{Hm}^{3}$, que se llena fundamentalmente con aguas invernales del río Cea, mediante el uso de infraestructuras existentes de transporte, como es el Canal de Trasvase Esla-Carrión. Se trata de mostrar una forma de disponer de recursos hídricos en una zona alejada de ríos que 
puedan aportar estos recursos, mediante balsas fuera de cauce, poniendo de manifiesto las ventajas e inconvenientes que este tipo de soluciones tienen.

Además, en el presente trabajo se expone una fórmula de colaboración entre la Administración y la Comunidad de Regantes creada para gestionar esta zona de riego, de forma que se atienden las necesidades en materia de seguridad de presas y balsas de esta singular regulación.

\section{2.- Descripción del tema y del proceso de ejecución del Trabajo.}

\section{1.- La importancia del regadío en Castilla y León}

La importancia que tiene el regadío sobre la población y la vertebración del territorio es sobradamente conocida. En Castilla y León el sector agrario representa prácticamente el doble de su valor en España y casi cuatro veces más que el valor que este sector tiene en la UE. El peso del sector agrario en nuestra comunidad se encuentra en torno al 5,5\% del VAB regional y si le añadimos el peso de la industria alimentaria regional alcanza en su conjunto un valor del $10 \%$ del VAB regional, dando trabajo a más de 100.000 personas en la región.

La agricultura regional es bastante sensible a las variaciones meteorológicas anuales. Las producciones de los cultivos en Castilla y León pueden llegar a tener variaciones medias de hasta el $25 \%$, debido a la fluctuación de las condiciones meteorológicas, y sobre todo al régimen de lluvias. Esto hace que las explotaciones agrícolas de secano tengan una dependencia extrema del medio. El regadío contribuye a reducir este problema, ya que reduce la dependencia de estos factores, incrementando las posibilidades de la alternativa y permitiendo aumentar las garantías en la consecución de los márgenes de las explotaciones.

Los datos oficiales del Ministerio de Agricultura y Ganadería contenidos en la Encuesta sobre superficies y rendimientos del año 2018, establecen una superficie regada en Castilla y León de 448.485 hectáreas.

Tabla 1.- Encuesta sobre superficies y rendimientos. Año 2018. MAPA

\begin{tabular}{|c|c|c|c|c|c|}
\hline $\begin{array}{l}\text { Comunidad } \\
\text { Autónoma }\end{array}$ & $\begin{array}{l}\text { Superficie } \\
\text { regadío (ha) }\end{array}$ & $\begin{array}{l}\text { Total, superficie } \\
\text { de cultivo }\end{array}$ & $\begin{array}{l}\text { Superficie } \\
\text { Geográfica (ha) }\end{array}$ & $\begin{array}{l}\text { Sup. } \\
\text { Regadío/Sup. } \\
\text { Cultivo (\%) }\end{array}$ & $\begin{array}{l}\text { Sup. } \\
\text { Regadío/Sup } \\
\text { Total (\%) }\end{array}$ \\
\hline Galicia & 16.871 & 369.077 & 2.957 .557 & 4,57 & 0,57 \\
\hline P. de Asturias & 483 & 24.954 & 1.060 .354 & 1,94 & 0,05 \\
\hline Cantabria & 424 & 6.987 & 532.944 & 6,07 & 0,08 \\
\hline País Vasco & 8.041 & 82.510 & 723.296 & 9,75 & 1,11 \\
\hline Navarra & 101.401 & 329.004 & 1.039 .038 & 30,82 & 9,76 \\
\hline La Rioja & 41.769 & 157.542 & 504.503 & 26,51 & 8,28 \\
\hline Aragón & 408.757 & 1.791 .074 & 4.772 .040 & 22,82 & 8,57 \\
\hline Cataluña & 260.601 & 822.681 & 3.210 .613 & 31,68 & 8,12 \\
\hline Baleares & 19.625 & 166.301 & 499.170 & 11,80 & 3,93 \\
\hline Castilla y León & 448.485 & 3.557 .687 & 9.422 .372 & 12,61 & 4,76 \\
\hline Madrid & 20.187 & 207.181 & 802.749 & 9,74 & 2,51 \\
\hline Castilla La Mancha & 553.888 & 3.709 .967 & 7.946 .100 & 14,93 & 6,97 \\
\hline C. Valenciana & 290.720 & 643.855 & 2.325 .913 & 45,15 & 12,50 \\
\hline R. de Murcia & 183.517 & 474.640 & 1.131 .387 & 38,66 & 16,22 \\
\hline Extremadura & 280.857 & 1.077 .728 & 4.163 .442 & 26,06 & 6,75 \\
\hline Andalucía & 1.048 .617 & 3.548 .596 & 8.758 .808 & 29,55 & 11,97 \\
\hline Canarias & 19.498 & 43.907 & 744.510 & 44,41 & 2,62 \\
\hline TOTAL & 3.703 .741 & 17.013 .691 & 50.594 .796 & 21,77 & 7,32 \\
\hline
\end{tabular}

Esto significa que Castilla y León dispone de un $12,10 \%$ de la superficie total regada de Estaña, siendo la tercera Comunidad Autónoma en cuanto a esta superficie, después de Andalucía $(28,31 \%)$ y Castilla La Mancha $(14,95 \%)$. 
Sin embargo, la superficie de regadío es solamente el $12,61 \%$ de la superficie que ocupan los cultivos, cifra inferior al resto de Comunidades autónomas, a excepción de las del norte de España, que, por sus condiciones climáticas, precisan del regadío en menor medida para obtener sus producciones agrícolas.

La creación de nuevos regadíos o la modernización de los mismos, son tradicionalmente una de las medidas más eficaces en la mejora de la competitividad de las explotaciones y por lo tanto del desarrollo rural, debido a las siguientes razones:

- Aumento y estabilización de la producción, lo que evita los desajustes y mejora la renta las explotaciones. Una hectárea de regadío genera un valor agregado bruto 3,5 veces superior al de una hectárea de secano. Los nuevos regadíos, asimismo, permiten diversificar la producción.

- Desde la perspectiva del desarrollo rural, la aportación del regadío es fundamental.

Primero, porque su potencial de trabajo triplica al del secano, contribuyendo con ello al mantenimiento de la población en el medio rural. La densidad de población es hasta 3 veces superior en zonas de regadío de alta intensidad respecto a zonas de secano. Las zonas de regadío presentan unas tasas de actividad muy superiores. El número de trabajadores es 3,6 veces superior (Figura 1).

El número de incorporaciones de jóvenes agricultores es 6,5 veces superior en zonas de alta intensidad de riego, que en zonas de secano (Figura 1).

La inversión total sujeta a ayudas de modernización es 7,5 veces superior a la que se produce en las zonas de secano (Figura 1).
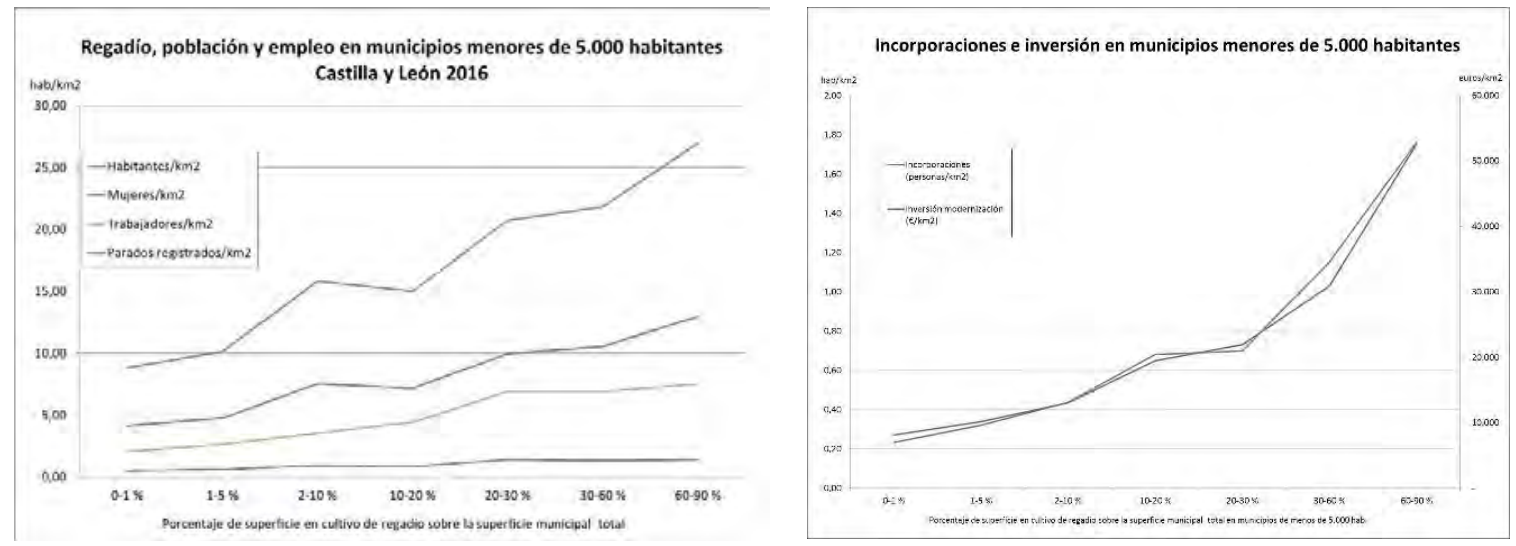

Figura 1. Efectos del regadío sobre la población, el empleo la incorporación de jóvenes y la inversión en municipios de menos de 5.000 habitantes en Castilla y León. Indicadores del Mapa de Infraestructuras de Castilla y León. Consejería de Agricultura y Ganadería 2016.

Segundo, porque favorece la generación de sinergias al potenciar muchas otras actividades en este medio. Así la industria agroalimentaria asociada a las producciones de regadío es una fuente de riqueza, empleo y desarrollo fundamental en muchas zonas rurales. La necesidad de adaptar a los mercados las producciones y la conveniencia de consolidar el sistema agroalimentario se consideran objetivos fundamentales a los que el regadío contribuye intensamente. 
- En el caso de las modernizaciones, se estima una reducción de los costes energéticos en más de un $50 \%$, al pasar de utilizar motores de gasoil a un sistema comunitario a través de una estación de bombeo moderna con rendimientos muy altos.

- Reducción de los costes de producción, concretamente en un menor uso de fertilizantes al evitarse pérdidas por lavado de los nitratos con motivo de un uso adecuado de las cantidades de agua. Esto en la práctica se traduce en ahorros de entre un 25 y un $30 \%$ en el abonado nitrogenado para obtener las producciones máximas. El coste de riego por aspersión en una zona modernizada es un 33,83\% inferior al coste del riego por aspersión en una zona no modernizada.

- Contribuyen a la sostenibilidad ambiental, pues reducen considerablemente el uso de agua con cifras medias del $20-25 \%$, permitiendo una adecuada gestión de los recursos naturales renovables de que se dispone. La producción en el futuro deberá tener la consideración de sostenible, y los regadíos modernos permiten realizar la actividad de forma competitiva para las explotaciones y respetando los valores ambientales del medio rural en el que se desarrolla. Además, este ahorro se produce al mismo tiempo que se intensifica la alternativa con cultivos de verano de alta demanda debido a una mayor eficiencia de distribución y aplicación.

- El número de incorporaciones de jóvenes en zonas modernizadas es un 80 \% superior respecto a zonas de regadío no modernizadas. La inversión particular de los agricultores en zonas modernizadas es un $240 \%$ superior a zonas no modernizadas.

Debido, en consecuencia, a que los regadíos se constituyen como una de las medidas más eficaces para la generación de actividad, el mantenimiento de la población y la creación de empleo en el medio rural, las inversiones de regadío se siguen considerando prioritarias en este nuevo periodo de programación en la Comunidad Autónoma.

\section{2.- La necesidad de incrementar la capacidad de regulación}

En los últimos años hemos podido comprobar como la situación de déficit de recursos disponibles es una situación que puede extenderse al conjunto de la cuenca, y que las consecuencias de lo que se denomina cambio climático hacen necesario seguir actuando para poder hacer frente de la mejor manera posible a este fenómeno adverso. La realidad es que las condiciones climáticas, acentuadas por este cambio climático, hacen absolutamente imprescindible poder disponer de los recursos en los momentos en los que se precisan, siendo totalmente necesarios para el mantenimiento de la actividad socioeconómica de nuestro territorio, del empleo y la población en el medio rural, y constituyéndose como elemento esencial en la vertebración de nuestro extenso y despoblado territorio.

Si se hace un análisis de la regulación en esta cuenca, comparativamente con el resto de las cuencas de España, el resultado puede reflejarse en el siguiente gráfico, obtenido con datos reflejados en las planificaciones hidrológicas de las diferentes cuencas (Figura 2): 


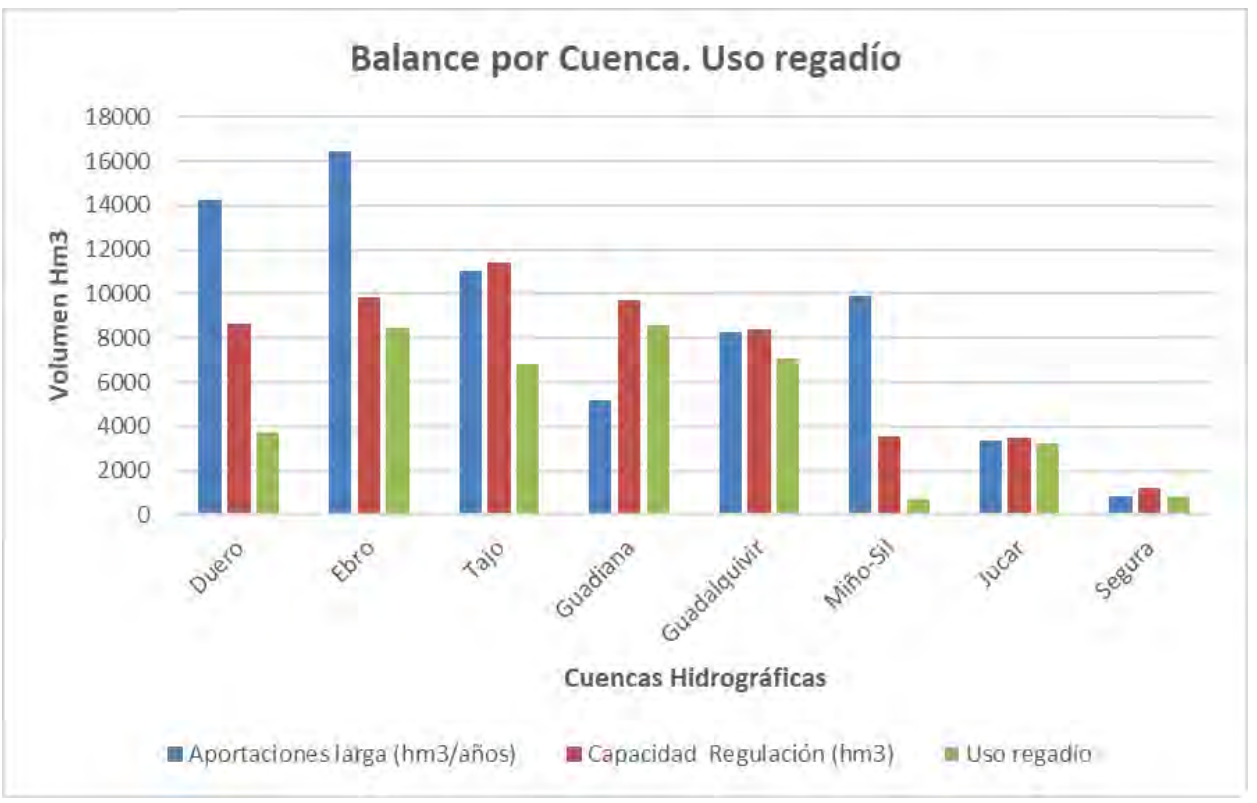

Figura 2. Comparación de la capacidad de regulación para diferentes cuencas de España. Elaboración propia con datos del Estudio "Posibilidades de aumento de recursos hídricos disponibles en Castilla y León”. Universidad de Burgos. 2019

Este gráfico pone de manifiesto los siguiente:

$\left.1^{\circ}\right)$ La cuenca del Duero es una de las que más aportaciones tiene, la segunda por debajo de la del Ebro, pero su capacidad total de regulación es prácticamente la mitad de las aportaciones medias. Resulta evidente que este déficit es una dificultad para atender las necesidades.

$2^{\circ}$ ) En la mayoría de las cuencas de España la capacidad de regulación es superior a las aportaciones, a excepción del Ebro, el Duero y el Miño. Esto es especialmente notable en algunas como el Guadiana (186\%) y en el Segura (176\%), si bien en esta última las aportaciones son muy reducidas. Estas, donde la capacidad es prácticamente el doble que las aportaciones, son las únicas cuencas que pueden tener capacidad de regulación hiperanual, algo que en el caso del Duero está muy lejano.

$3^{\circ}$ ) Si se profundiza un poco más en la regulación del Duero, a diferencia de otras cuencas, además una buena parte de esta regulación está aguas abajo de los posibles usos. Los embalses que se sitúan en la frontera con Portugal (Almendra, Ricobayo, Saucelle, Aldeadávila y Castro), suponen una capacidad de regulación de más de $4.100 \mathrm{Hm} 3$, que es un $48 \%$ de la capacidad total de regulación de la cuenca del Duero. Estas regulaciones están, como se ha indicado aguas abajo de los posibles usos en la cuenca, por lo que no pueden tener prácticamente otro aprovechamiento que el hidroeléctrico, no pudiéndose destinar a usos consuntivos en nuestro territorio. Esta diferencia es muy importante entre el caso del Duero y el resto de las cuencas, pues reduce realmente la capacidad de regulación a estos efectos a menos de $4.000 \mathrm{hm} 3$. Esta situación coloca a la cuenca del Duero en cifras y posibilidades de aprovechamiento de los recursos regulados muy lejanos a cualquier otra cuenca de España. A tenor de estos datos, la cuenca del Duero es la que menos aprovechamientos regulados tiene de España.

En la situación actual, y dado que los hechos nos están mostrando que, además de la reducción de la precipitación y las aportaciones, el cambio climático trae como consecuencia un incremento de los fenómenos extremos, donde se producirán épocas de exceso de precipitaciones más intensas de lo normal y épocas de sequías, también más acentuadas de lo normal, parece absolutamente necesario que se contemplen estas actuaciones, si no queremos sufrir con el máximo rigor las consecuencias del cambio climático. 
Se trata de una situación que hay que plantear como crítica en las condiciones españolas, con especial gravedad en el Duero, dada la escasez de capacidad de regulación, que debe llevar a plantear soluciones totalmente estratégicas, y si se quiere fuera de los cauces habituales, como corresponde a situaciones de absoluta excepcionalidad.

\section{3.- La balsa del trasvase Esla-Carrión. Antecedentes}

Como ejemplo de una posibilidad de regulación de recursos invernales, se muestra la zona regable del Sector IV del Canal Esla Carrión, para cuyo desarrollo ha sido necesaria la construcción de una balsa fuera de cauce.

El desarrollo de esta zona regable parte de los compromisos teóricamente adquiridos por la Confederación Hidrográfica del Duero cuando se ejecutaron las obras del Canal de Trasvase desde el Esla al Carrión, como solución provisional para aportar recursos adicionales a las zonas regables dependientes del río Carrión, como consecuencia de no ejecutar la presa de Vidrieros. La construcción del Canal incluía, entre las condiciones de expropiación, la dotación de agua a los municipios afectados, para la transformación en regadío de una superficie proporcional a la superficie expropiada.

Por Acuerdo de la Junta de Castilla y León 18/2003 de 30 de enero (B.O.C. y L. nº 24 de 5 de febrero), se declara la utilidad pública y urgente ejecución de las mejoras y obras a ejecutar en la Zona Regable del Esla Carrión, en las provincias de León, Palencia y Valladolid, estableciendo que las obras a ejecutar en los municipios de Sahagún en León, Fontihoyuelo, Herrín de Campos, Melgar de Arriba, Santervás de Campos, Villacarralón, Villafrades y Villalón de Campos en Valladolid y Autillo de Campos, Boadilla de Rioseco, Frechilla, Fuentes de Nava y Guaza de Campos en Palencia, deberán estar incluidas en el correspondiente Plan de Mejoras Territoriales y Obras redactado por la Consejería de Agricultura y Ganadería.

Con el fin de dotar de agua a las zonas con concesión, se redactó por parte de la Dirección General de Desarrollo Rural de la Junta de Castilla y León el Plan de Mejoras Territoriales y Obras, que fue aprobado por Orden AYG/723/2003 de 19 de mayo de 2003 (BOC y L de Jueves 5 de junio de 2.003) y posteriormente modificado por Orden AY/1330/2003 de 16 de octubre de 2003.

En este documento se recoge una sectorización de la zona regable en cinco sectores, dando el nombre de Sector IV al que nos ocupa. Los cinco sectores son los siguientes (se reflejan en el plano que se acompaña a continuación, Figura 3): 

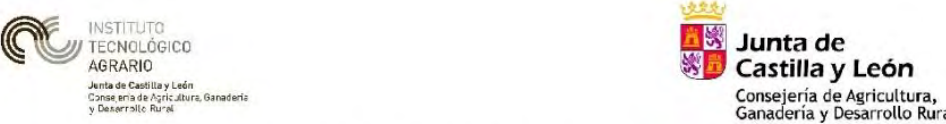

\section{TRANSFORMACIÓN EN REGADÍO SECTOR IV TRASVASE ESLA-CARRIÓN}

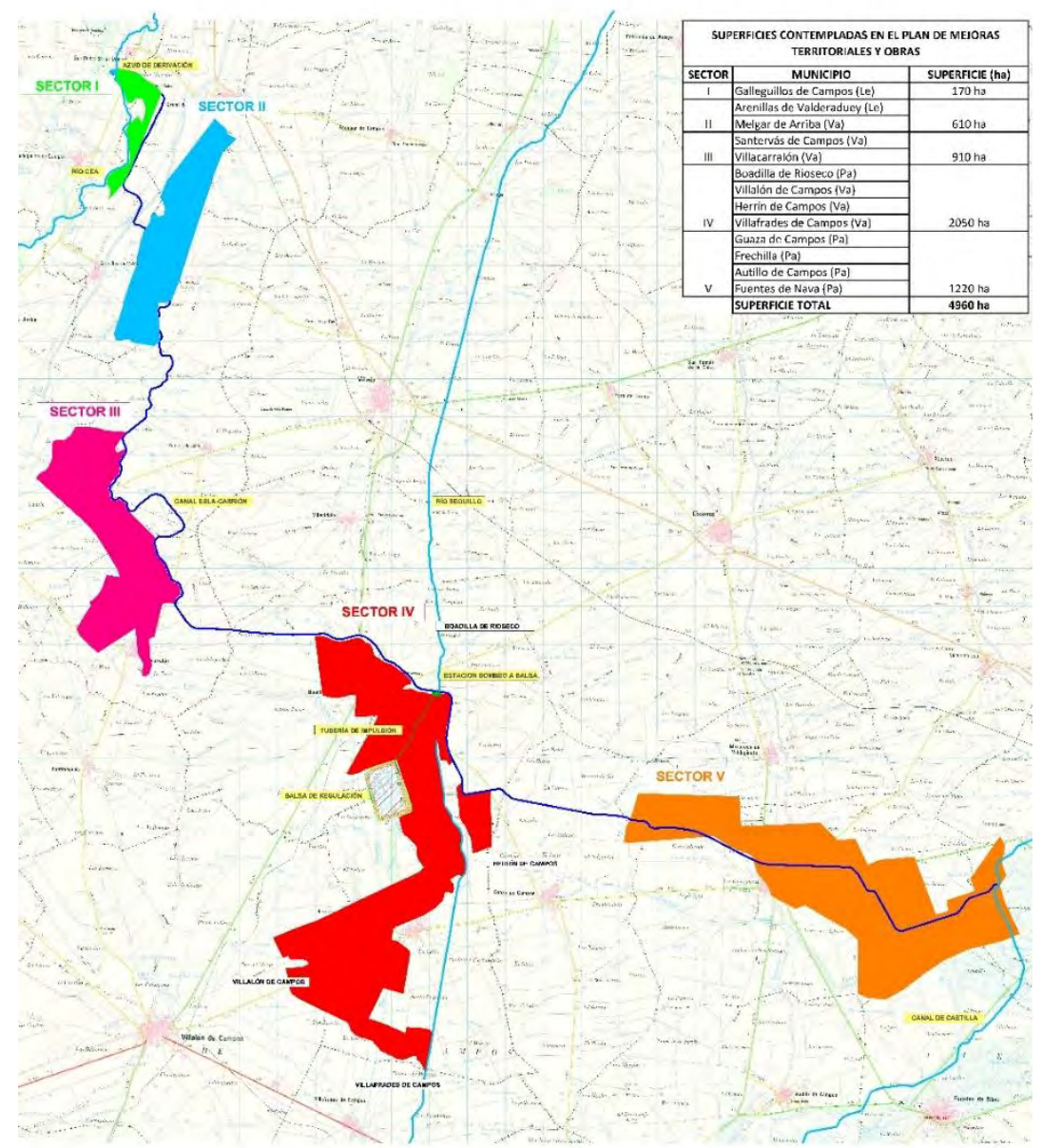

Figura 3. Planificación de los regadíos de la zona regable del Esla-Carrión. Elaboración propia.

La superficie comprometida por la Confederación como consecuencia de las obras del Canal en esta zona fue de 472 hectáreas. La causa de este desfase entre las superficies concedidas y las que se pretenden poner en regadío, es que, con posterioridad a las superficies concedidas por confederación, se detectó un gran interés por aumentar la zona regable en Herrín de Campos y extender su área de influencia a los vecinos Villalón y Villafrades de Campos. Ante esta situación se procedió en el plan de mejoras territoriales y obras a proponer una balsa que permitiera hacer frente a las superficies demandadas, almacenando aguas invernales, sin comprometer los consumos estivales concedidos por el canal.

En el Plan de Mejoras se hace referencia a la concesión inicialmente prevista de $400 \mathrm{l} / \mathrm{s}$, en función de los compromisos expropiatorios, suficientes para las demandas inicialmente previstas, pero no para la demanda real de riego, por ello y para hacer frente a la misma, se proyecta una balsa de acumulación de $10 \mathrm{Hm} 3$ que pretende llenarse con aguas invernales transportadas por el canal y procedentes del río Cea.

Posteriormente en marzo de 2004 por parte de Técnicos del Instituto Tecnológico Agrario de Castilla y León se procede a redactar el Proyecto Básico de Transformación en Regadío de 
la Zona en cuestión con el fin de determinar con más verosimilitud los costes reales de la inversión y además tramitar la concesión de aguas. Para ello, en dicho documento, se recoge como propuesta el adelanto de la campaña de desembalse del Canal del trasvase en 70 días, respecto a la inicialmente prevista (el 1 de abril), bombeando $2.400 \mathrm{l} / \mathrm{s}$ hasta esa fecha en que comienza la campaña de riegos por parte de la Confederación Hidrográfica del Duero. A partir de esa fecha, permitir extraer la concesión comprometida original de $400 \mathrm{l} . / \mathrm{s}$. De esta forma la concesión permitiría llenar la balsa y satisfacer las demandas reales de la zona regable que nos ocupa.

\section{4.- Proceso de concentración parcelaria}

Además, se ha realizado por la Dirección General de Producción Agropecuaria y Desarrollo Rural, la Concentración Parcelaria de la Zona, en aplicación del Artículo 71 de la ley 14/1990, por Orden AyG/1508/2003 de 17 de noviembre (BOC y $L$ de 26 de noviembre de 2003). Las Bases Definitivas fueron aprobadas con fecha el 22 de julio de 2005. El Acuerdo de Concentración se aprobó el 16 de julio de 2009 y la toma de posesión se realizó el 16 de octubre de 2010.

El proceso ha comprendido una superficie total de 16.296 ha correspondientes a 1260 propietarios en la totalidad de los cuatro términos municipales incluidos en la actuación. El hecho más importante es que de 4.759 parcelas aportadas inicialmente por los propietarios se han pasado a 2310 fincas de reemplazo adjudicadas, lo que supone la reducción en un 48 $\%$ el número de parcelas entregadas.

Tabla 2.- Resumen del resultado de la concentración parcelaria. Elaboración propia con datos del Acuerdo de Concentración, Julio de 2009.

\begin{tabular}{|c|c|c|}
\hline $\begin{array}{l}\text { CONCLUSIONES DEL PROCESO DE } \\
\text { CONCENTRACIÓN PARCELARIA }\end{array}$ & ANTES & $\begin{array}{l}\text { DESPUÉS } \\
\text { (PROYECTO) }\end{array}$ \\
\hline No TOTAL DE PARCELAS & 4.759 & 2.310 \\
\hline NN PARCELAS POR PROPIETARIO & 3,76 & 1,83 \\
\hline SUPERFICIE MEDIA DE LAS PARCELAS & 3-37-38 ha & $6-87-33$ ha \\
\hline
\end{tabular}

En este momento, en virtud de la Ley $1 / 2014$, de 19 de marzo, Agraria de Castilla y León, todas las actuaciones financiadas por la Comunidad Autónoma en materia de regadíos, ya sea modernización de regadíos, ya sea nueva transformación han de ir complementadas con un proceso de concentración parcelaria.

\section{5.- Desarrollo de las obras en la zona}

Para poder ejecutar las obras y tramitar la correspondiente concesión de aguas, se procedió a la tramitación ambiental de la actuación, sometiendo a trámite de impacto ambiental las obras incluidas en el Plan de Obras y Mejoras Territoriales del Sector IV, siendo publicada la declaración de Impacto Ambiental del mismo por Resolución de 23 de octubre de 2003 (BOC y $L$ de 31 de Octubre de 2003), sobre los proyectos de concentración parcelaría y transformación en regadío del Sector IV de la Zona Regable del Canal Esla Carrión, en los términos municipales de Villalón de Campos, Villafrades de Campos y Herrín de Campos en 
la provincia de Valladolid y Boadilla de Rioseco en Palencia, promovido por la Consejería de Agricultura y Ganadería de la Junta de Castilla y León

Respecto al otorgamiento de la concesión, ésta se solicitó con fecha 23 de marzo de 2004 . Con fecha 4 de mayo de 2004 se publicó en el Boletín Oficial de la Provincia de Valladolid anuncio sobre la petición de concesión para la superficie de 2050 hectáreas, correspondiente a este sector. El mismo anuncio se publicó en el Boletín Oficial de la Provincia de Palencia el 10 de mayo de 2004, al existir superficie de transformación en las dos provincias.

Los proyectos, en el marco del trámite correspondiente, fueron enviados a la Confederación Hidrográfica del Duero con fecha 6 de mayo de 2004.

Después de una discusión importante sobre la validez de la declaración de impacto ambiental que publicó la Junta de Castilla y León, respecto al trámite de otorgamiento de la concesión, por parte de la Confederación Hidrográfica del Duero, que demoró este trámite varios años, con fecha 23 de marzo de 2012 se otorgó una concesión de aguas a la Dirección General de Producción Agropecuaria y Desarrollo Rural, para el riego de 2.050 hectáreas, con un volumen máximo anual de $10 \mathrm{Hm} 3$, con aguas procedentes del río Cea y del río Esla, a trasladar por el canal Cea Carrión. Las aguas se derivarán del río Cea entre el 1 de noviembre y el 31 de marzo, en un periodo no superior a 90 días, y del río Esla entre el 1 de abril y el 30 de septiembre de cada año.

Las obras ejecutadas y en ejecución en la zona y su estado actual se distribuyen en varios proyectos, que son los siguientes:

$\left.1^{\circ}\right)$ Proyecto de "Infraestructura rural de la zona de concentración parcelaria de Herrín, Villafrades, Villalón y Boadilla de Rioseco -Fase I- (Valladolid-Palencia)": Ejecutadas en su totalidad, incluían la ejecución de las siguientes actuaciones más importantes: construcción de una red de caminos de $343 \mathrm{~km}$ de longitud, todos ellos estabilizados con zahorra natural, ejecución de una red de desagües de $295 \mathrm{~km}$ de longitud, realización de 689 obras de fábrica tipo caño de diferentes diámetros, así como 5.030 $\mathrm{m}^{3}$ de escollera de protección de márgenes de arroyos. La inversión total de esta obra alcanzó 10.389.837,06, finalizando en el año 2015.

$2^{\circ}$ ) Proyecto de "TRANSFORMACIÓN EN REGADÍO DEL SECTOR IV DE LA ZONA REGABLE DEL TRASVASE ESLA-CARRIÓN. OBRAS DE INTERÉS GENERAL (VALLADOLID-PALENCIA)". Estas obras finalizaron en el año 2015, por importe de 22.898.411,30€, IVA incluido. La obra consiste en la ejecución de una captación sobre el canal del Trasvase Esla-Carrión, y conducción hacia una estación de bombeo con la instalación de equipos de desbaste y filtrado automático. La estación de bombeo impulsa el agua a una presión de 1,9 bares y un caudal variable entre 400 y $2400 \mathrm{l} / \mathrm{s}$ función del volumen que ponga a disposición la Confederación Hidrográfica del Duero.

DOI:10.31428/10317/8716 
La impulsión tiene una longitud de 2.410 metros en tubería de hormigón con camisa de chapa hasta una balsa de materiales sueltos de regulación anual con una capacidad máxima de 9,8 Hm3 impermeabilizada con arcilla procedente de la excavación, protección de dique con piedra escollera, altura de lámina de agua de $10 \mathrm{~m}$ y superficie ocupada en planta de 117 ha. Adicionalmente se instalan sistemas redundantes de seguridad para el llenado y vaciado, la auscultación de la balsa, así como de comunicación para el control en tiempo real vía web de su estado. Incluye la restauración ambiental del medio natural de la zona, con la ejecución de un observatorio de visualización de aves, plantaciones arbóreas y arbustivas y seguimiento arqueológico y ambiental.

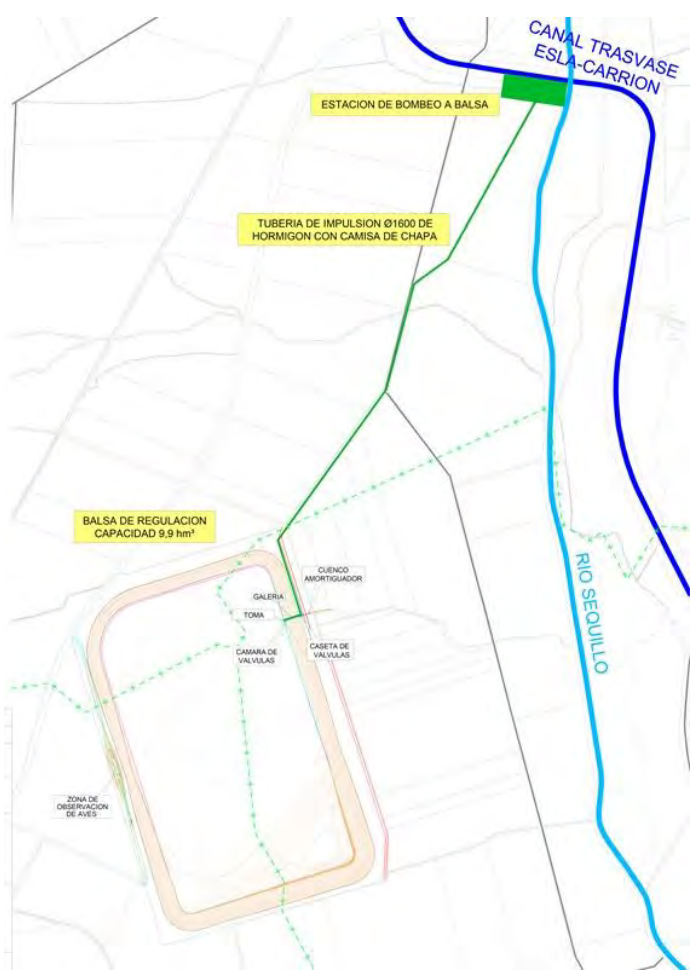

Figura 4. Diseño del llenado de la balsa.

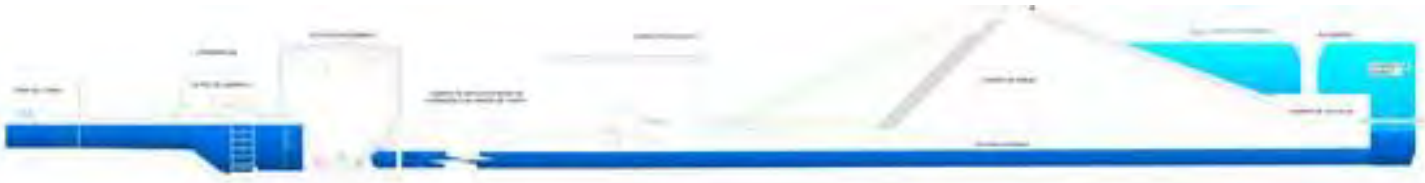

Figura 5. Sección de infraestructuras de llenado de la balsa.

\section{SECCION ESTACION DE BOMBEO}

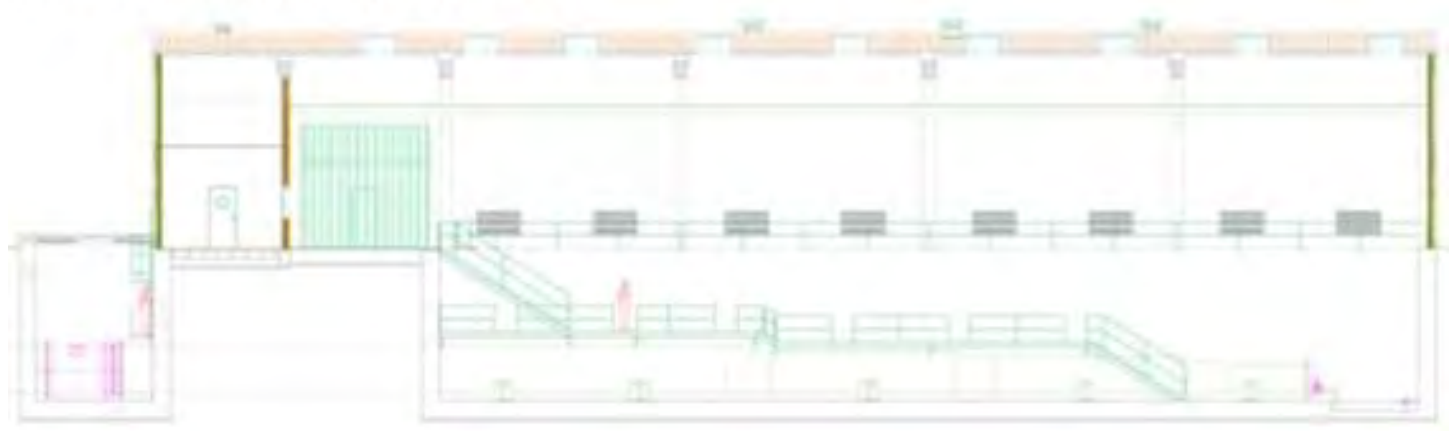

Figura 6. Sección de la estación de bombeo.

La balsa incluida en el proyecto tiene la siguiente sección tipo: 


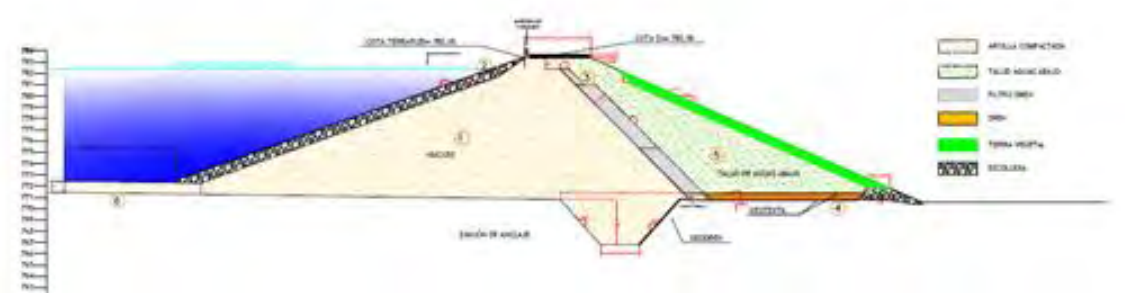

Figura 7. Sección tipo de la balsa.

Como se observa en la imagen está compuesta por un repie de escollera de protección contra el oleaje, un núcleo (que engloba el clásico espaldón interior y el núcleo de una sección heterogénea de materiales sueltos), un filtro dren tipo sherard unido a un manto de grava y un talud exterior compuesto por los materiales menos impermeables del vaso.

La mayor complejidad de esta balsa debido a su extensión es el control de materiales del núcleo y evitar la comunicación de materiales filtrantes con el exterior de la balsa.

La zona en la que se sitúa la balsa está compuesta por materiales que van desde arcillas de plasticidad media a arenas pasando por arcillas limosas, limos arenosos y arenas limosas. El estudio geotécnico inicial consistió en realizar 6 sondeos en el eje y una malla de calicatas de $100^{\star} 100 \mathrm{~m}$ para poder definir un plano en 3 dimensiones de las capas subhorizontales de cada uno de los materiales existentes. Con esta información se realizó un diseño previo que se fue complementando con zanjas de dos a tres metros de profundidad que servían para corregir el modelo inicial.

Una vez realizado el análisis geotécnico de las zanjas se definieron las zonas y espesores de los materiales utilizables para la formación del núcleo y del talud exterior y se vigiló tanto la procedencia del material como la compactación del mismo durante todo el horario de actividad de la maquinaria.

El otro punto crítico de control era el terreno situado bajo el zanjón de anclaje, puntualmente teníamos información con los sondeos, pero es insuficiente para conocer longitudinalmente el eje del zanjón por lo que se optó por realizar una tomografía correlacionada con los sondeos. A continuación, se incluyen tres perfiles obtenidos de la tomografía.

En el primero se observa que hay arenas con una continuidad hacia el exterior de la balsa y al excavar el zanjón se corroboró la presencia de esas arenas por lo que se ejecutó una pantalla de bentonita en esa zona. En el resto de las secciones se ven colores azules o verdes que se corresponden con arcillas de baja a media plasticidad y pequeñas zonas de arenas y limos que se impermeabilizaron aumentando ligeramente la profundidad del zanjón de anclaje.

\section{$3^{\circ}$ ) Proyecto de "REDES DE}

DISTRIBUCIÓN DEL SECTOR IV DE

LA ZONA REGABLE DEL TRASVASE

ESLA-CAMPOS". El proyecto al igual que las actuaciones de las obras de interés general ha sido redactado por el ITACyL y está siendo dirigido por 


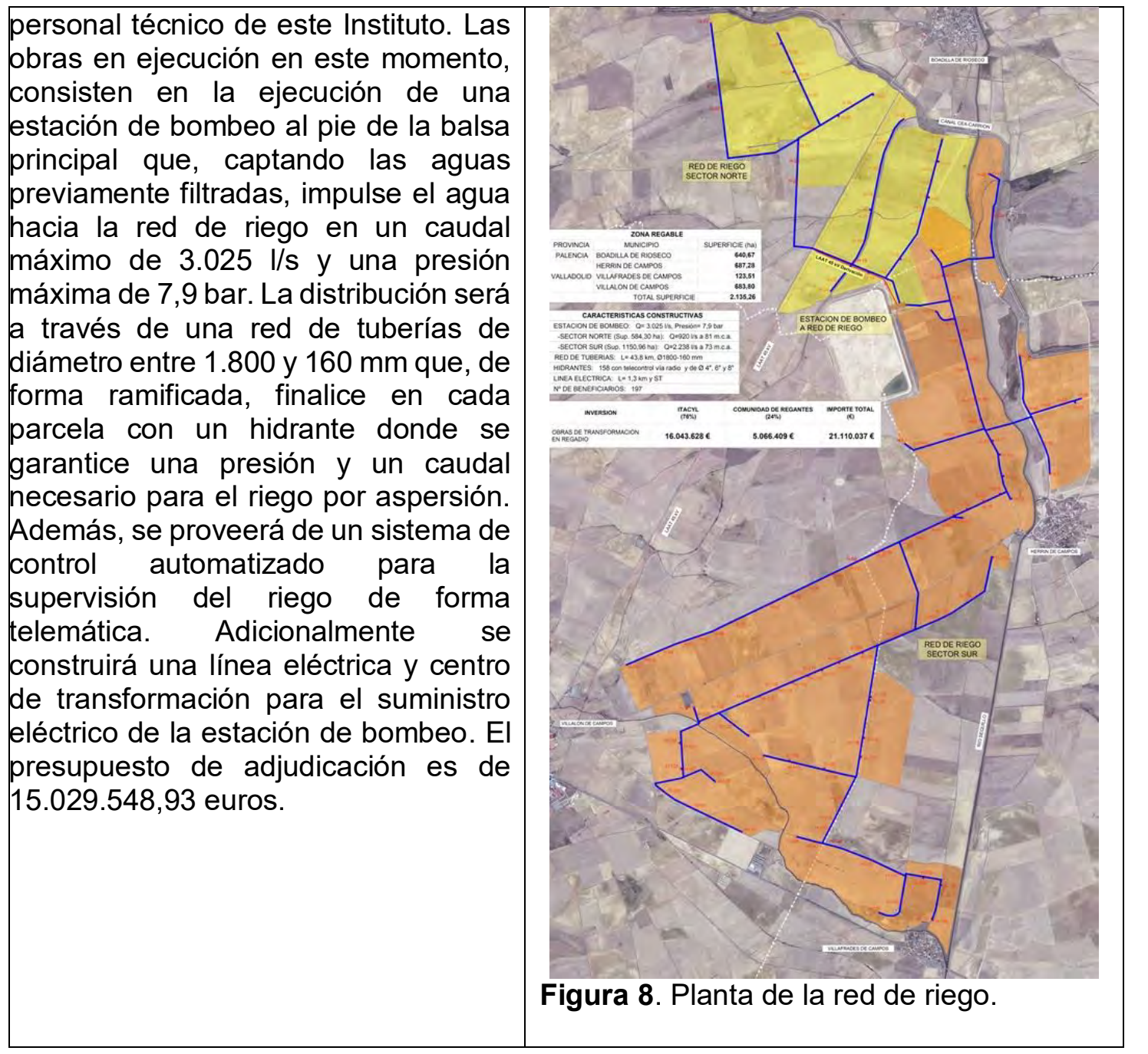

\section{6.- Cumplimiento de las obligaciones en materia de seguridad de presas y balsas.}

Se trata de una balsa de materiales sueltos con una tipología encuadrada entre las presas homogéneas y heterogéneas para poder aprovechar los materiales del vaso. La altura máxima de los taludes es de 15 metros y la superficie de ocupación total es de aproximadamente 120 ha.

De acuerdo con el artículo 358.a) del Real Decreto 9/2008, de 11 de enero, por el que se modifica el Reglamento del Dominio Público Hidráulico ésta balsa se clasifica como gran presa. Según el artículo 367.1 de este mismo Reglamento, el titular está obligado a solicitar su clasificación y registro. Por lo anterior, desde el ITACyL se redactó la propuesta de clasificación de la balsa en función del riesgo potencial que pueda derivarse de su posible rotura siendo clasificada como Categoría $\mathrm{A}$.

Para dar cumplimiento a lo establecido en el artículo 5.7 del Reglamento Técnico sobre Seguridad de Presas y Embalses (1.996) se redactan las Normas de Explotación de la balsa que son aprobadas por la Dirección General del Agua. 
Según la Directriz Básica de Planificación de Protección Civil también es obligatoria la redacción de un Plan de Emergencia que fue aprobado por la Dirección General del Agua.

Aprobado el Plan, el titular de la balsa dispone de dos años para implantarlo. En el caso de la balsa que nos ocupa la implantación ha finalizado 10 de marzo de 2020 y tiene la particularidad de que se emplea un Centro de Gestión de Emergencia Móvil (figura 9) que, entre otras funciones, permite las comunicaciones con todos los organismos implicados en el plan de emergencia de la balsa y con los sistemas de aviso a la población, consistente, en este caso, en una sirena con 12+4 altavoces ubicada al pie de la balsa. También permite vigilar las instalaciones a través de las imágenes recogidas por las cámaras instaladas.
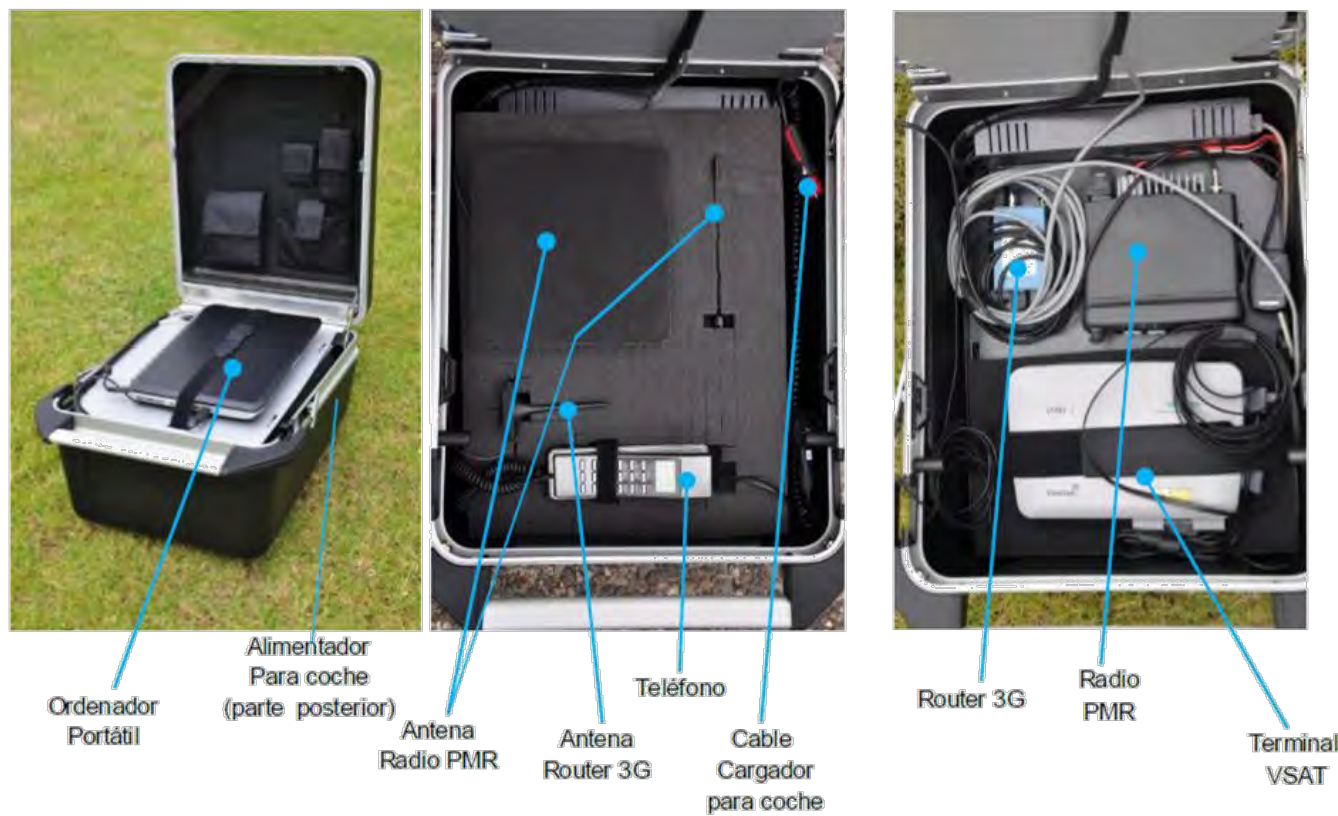

Figura 9. Centro de Gestión de Emergencia Móvil

Cuando el ITACyL acometió en el año 2013 la primera implantación de un Plan de Emergencia el reto que se planteó fue el de disponer de los mismos medios y herramientas de gestión y control que los titulares con mayores recursos, pero adaptados a su realidad, un número elevado de elementos (presas/balsas) a gestionar, que se encuentran en medio del campo, sin ninguna vigilancia, sometidas a actos vandálicos y con un presupuesto para su explotación y mantenimiento ajustado. Con el objetivo anterior como meta, se optó por recurrir a un Centro de Gestión de Emergencia Móvil para el desarrollo de las labores de explotación y emergencias que permite gestionar varias presas/balsas simultáneamente con una reducción de los costes de mantenimiento, explotación, licencias, actualización de equipos y gestión de la ciberseguridad.

En aquel momento, adoptar esta opción era bastante novedosa, aunque ya se recogía como alternativa en los acuerdos de la Comisión Nacional de Protección Civil. Con posterioridad, ha sido implantada en numerosas presas y balsas y en la Guía para la Implantación de Planes de emergencia de presas, publicada en 2017, se recoge esta alternativa siempre que integre el sistema de comunicaciones, el sistema de aviso a la población, y que disponga de toda la información relativa a la presa/balsa y a su Plan de Emergencia.

De esta manera, el Centro de Gestión de Emergencia Móvil, que nació como un mero gestor de emergencias, ha evolucionado hasta desarrollar una plataforma web que permite llevar una gestión integral de la explotación de diversas infraestructuras hidráulicas al implementarse, entre otras utilidades, el sistema de auscultación de la presa/balsa, el archivo técnico, el plan 
de mantenimiento y prealertas o la videovigilancia. En la figura 10 se muestra la interfaz y los módulos implementados en el sistema actual.

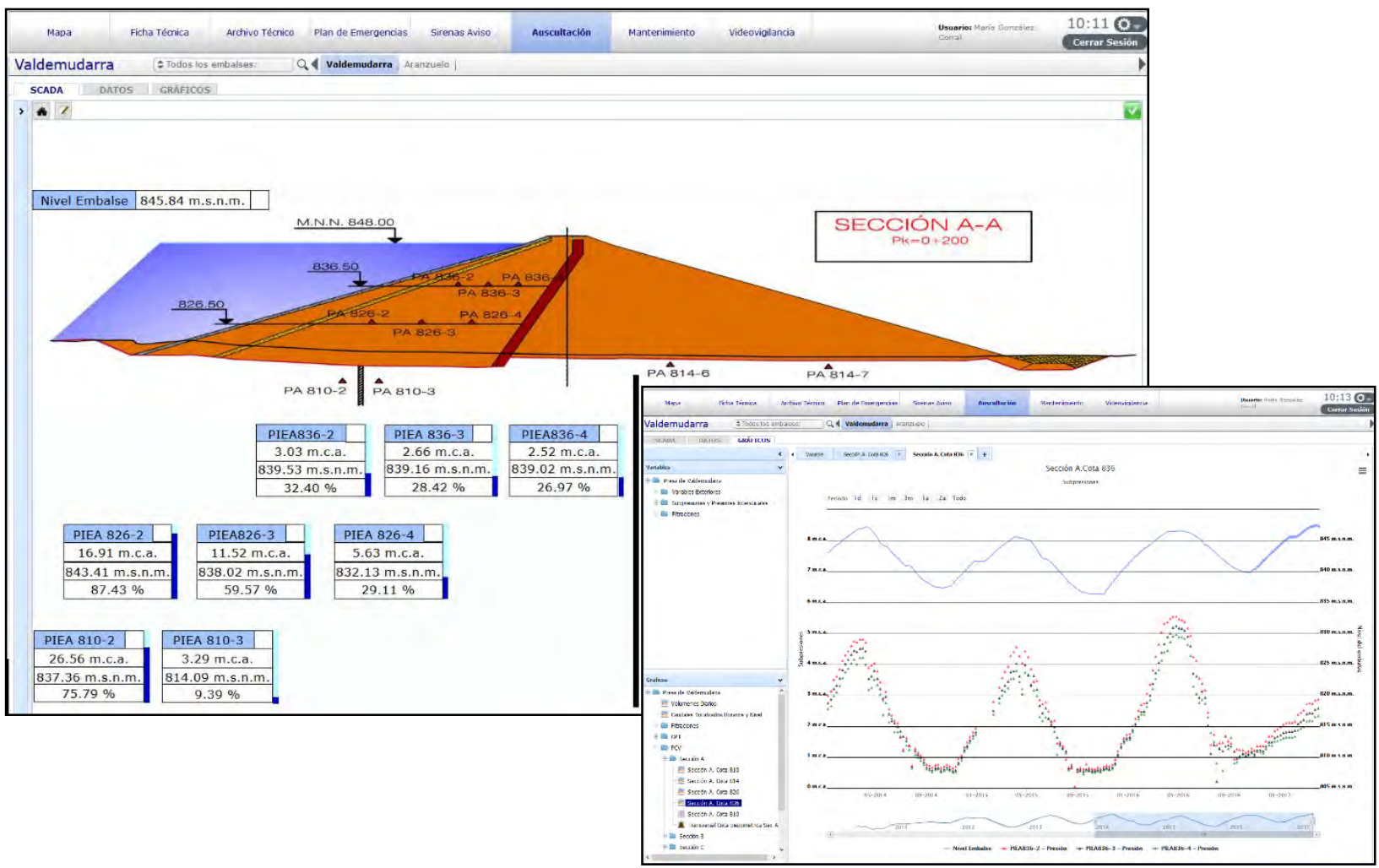

Figura 10. Interfaz del módulo de Auscultación y generación de gráficos (iDam by Lafcarr)

Finalizada la implantación, se ha iniciado en estos momentos la Puesta en Carga de la balsa. Para llevar a cabo esta fase el titular de la balsa designa a un técnico competente que es el encargado de redactar el Plan de Puesta en Carga y desarrollar sus contenidos. Cuando se dé por finaliza esta fase ya puede decirse que la balsa se encuentra en explotación y es cuando surge la figura del Director de Explotación y del Plan de Emergencia.

Hasta la fecha, todos estos trámites se realizan desde el ITACyL que como promotor de las obras es titular de la misma, pero el Instituto desarrolla todas estas infraestructuras para entregárselas a comunidades de regantes que pasan a ser titulares de las mismas y por tanto responsables del cumplimiento de la normativa vigente en materia de seguridad. No obstante, siendo de vital importancia garantizar la seguridad de las presas/balsas, por parte de la Junta de Castilla y León se consideró conveniente asumir la realización de determinados trabajos técnicos, como asunción voluntaria de una obligación que no es suya, pero que se enmarca dentro del interés general, previa firma de un convenio de colaboración entre las comunidades de regantes y el ITACyL.

En estos convenios quedan definidas las tareas que asumirán cada una de las partes y sus costes. Por parte de las Comunidades de Regantes asumirán los costes ordinarios de explotación y un canon a pagar al ITACyL. Mientras que el Instituto redactará proyectos, ejecutará las obras necesarias y asumirá la dirección de explotación y de los planes de emergencia.

Al gestionar el Instituto de manera conjunta un grupo de presas/balsas se consiguen abaratar los costes de desplazamientos y se comparten gastos de explotación e implantación de planes de emergencia. 
En la tabla 3 analizamos los costes de implantación del plan de emergencia de seis presas de categoría $\mathrm{A} / \mathrm{B}$, considerando que dos de ellas tienen dos elementos de aviso acústico a la población, otras dos sólo un elemento y las dos restantes ninguno.

Tabla 3. Cuadro comparativo costes implantación centro de gestión de emergencia móvil más centro de control en la nube vs sala de emergencia más centro de control fijo. Elaboración propia.

\begin{tabular}{|c|c|c|c|c|c|c|c|}
\hline & PROJ.1 & PROJ.2 & PROJ.3 & PROJ.4 & PROJ.5 & PROJ.6 & TOTAL \\
\hline & \multicolumn{7}{|c|}{ OPCIÓN SALA EMERGENCIA MOVIL A COMPARTIR PARA SEIS PRESAS } \\
\hline Aviso acústico & $35.000 €$ & $35.000 €$ & $15.000 €$ & $15.000 €$ & $0 €$ & $0 €$ & \\
\hline Sala móvil & $15.000 €$ & $2.500 €$ & $2.500 €$ & $2.500 €$ & $2.500 €$ & $2.500 €$ & \\
\hline C.Control Nube & $2.000 €$ & $2.000 €$ & $2.000 €$ & $2.000 €$ & $2.000 €$ & $2.000 €$ & \\
\hline \multirow[t]{2}{*}{ TOTAL } & $52.000 €$ & $39.500 €$ & $19.500 €$ & $19.500 €$ & $4.500 €$ & $4.500 €$ & $139.500 €$ \\
\hline & \multicolumn{7}{|c|}{ OPCIÓN SALA DE EMERGENCIA CONVENCIONAL } \\
\hline Aviso acústico & $35.000 €$ & $35.000 €$ & $15.000 €$ & $15.000 €$ & $0 €$ & $0 €$ & \\
\hline $\begin{array}{l}\text { Sala } \\
\text { emergencia }\end{array}$ & $40.000 €$ & $40.000 €$ & $40.000 €$ & $40.000 €$ & $40.000 €$ & $40.000 €$ & \\
\hline Cent. Control & $18.000 €$ & $3.500 €$ & $3.500 €$ & $3.500 €$ & $3.500 €$ & $3.500 €$ & \\
\hline TOTAL & $93.000 €$ & $78.500 €$ & $58.500 €$ & $58.500 €$ & $43.500 €$ & $43.500 €$ & $375.500 €$ \\
\hline
\end{tabular}

\section{3.- Conclusiones}

Como conclusiones de este trabajo pueden indicarse las siguientes:

$1^{\text {a) }}$ Los elementos estructurales de regulación, como esta macro balsa, construida fuera del cauce pueden ser una solución para regular recursos de invierno siempre que se den técnicamente las condiciones técnicas para ello, como son:

- Que exista un elemento de transporte en invierno de los recursos hídricos al que se asocien para su llenado. En este caso el Canal de Trasvase Esla Carrión que es una infraestructura construida que no tiene uso en invierno, cuando hay que llenar la balsa.

- Que en su ubicación existan materiales con la impermeabilidad adecuada para poder desarrollar su construcción, lo que permite que la inversión, aunque alta, no sea imposible de acometer.

Este planteamiento no habría sido posible sin la existencia de una canal, con una inversión ya desarrollada, y en un lugar como la tierra de campos, con unas características del terreno que lo permiten.

$\left.2^{a}\right)$ A pesar de darse las condiciones que hagan posible su planteamiento, son actuaciones técnicamente complejas, más caras y con un objetivo limitado en cuanto a la capacidad que aportan, por lo que su planteamiento ha de quedar circunscrito a la imposibilidad de regular los recursos de otra manera, como puede ser la construcción de una presa en cauce, el recrecimiento de infraestructuras existentes. No obstante, en la valoración de su coste habría que incorporar como aspecto positivo el hecho de que a una infraestructura ya ejecutada y cuya inversión se ha realizado completamente, se le pueda dar una mayor uso y aprovechamiento, lo que sin duda mejora la ratio coste/capacidad de regulación.

$\left.3^{a}\right)$ A pesar de estas dificultades, si se dan las condiciones adecuadas, pueden ser la única solución para desarrollar zonas regables de no mucha superficie, vinculadas a las mismas en determinadas zonas, como la que se plantea en este trabajo. En este sentido son de enorme interés para conseguir que los efectos sociales y económicos que el regadío tiene sobre el 
territorio rural se puedan extender por algunas de las zonas más afectadas por la despoblación y la falta de actividad.

$4^{\circ}$ ) Desde el punto de vista de la seguridad, son elementos importantes que requieren de atenciones adecuadas, por lo que hay que implementar los elementos de auscultación y control suficientes para su funcionamiento sin problemas.

$\left.5^{\circ}\right)$ Esta circunstancia hace que, como en el resto de los casos, el cumplimiento de las obligaciones exigidas por la normativa vigente en esta materia aconseje el apoyo público a los titulares de las mismas, las Comunidades de Regantes, para poder facilitarlas el trabajo técnico y el cumplimiento de las obligaciones relacionadas con la seguridad y el funcionamiento de las infraestructuras. Un ejemplo de esta colaboración que está resultando exitoso es el modelo de convenio que tiene el Instituto Tecnológico Agrario de Castilla y León para esta materia.

$\left.6^{\circ}\right)$ Para regulaciones de capacidad reducida es necesario buscar fórmulas para el cumplimiento de la normativa en materia de seguridad, que sin restar efectividad y garantizando en todo momento la seguridad que pretende la normativa, sean asumibles para los usuarios, y no incrementen de forma desproporcionada los costes. En este sentido, ideas asumidas por los órganos de control, como las salas de control móviles, el uso de medios compartidos para varias infraestructuras, son planteamientos que contribuyen de manera adecuada a lograr este objetivo. 